\title{
Evaluación de la capacidad estacional de utilizar eventos de precipitación en tres especies de arbustos nativos de Chile con distintos sistemas radiculares
}

\author{
Assessment of the seasonal ability to use precipitation events in three native Chilean \\ shrubs species with different root systems
}

\section{RAUL TORRES ${ }^{1}$, FRANCISCO A. SQUEO $^{1}$, CÁRMEN JORQUERA $^{2}$, EVELYN AGUIRRE $^{3} \&$ JAMES R. EHLERINGER $^{4}$}

\author{
${ }^{1}$ Departamento de Biología, Facultad de Ciencias, Universidad de La Serena, Casilla 599, \\ La Serena, Chile; e-mail: f_squeo@userena.cl \\ ${ }^{2}$ Departamento de Agronomía, Facultad de Ciencias, Universidad de La Serena, Casilla 599, \\ La Serena, Chile \\ ${ }^{3}$ Comisión Chilena de Energía Nuclear \\ ${ }^{4}$ University of Utah, Salt Lake City, Utah, U.S.A.
}

\begin{abstract}
RESUMEN
Se evaluó la capacidad estacional de utilizar un evento de precipitación en tres especies arbustivas con diferentes sistemas radiculares (dimórficos: Balbisia peduncularis, Senna cumingii; profundo: Haplopappus parvifolius) en la Quebrada El Romeral, norte-centro de Chile. El sitio posee un clima tipo mediterráneo árido con influencia de neblinas costeras y una precipitación promedio anual de $78 \mathrm{~mm}$ en los últimos 30 años. La utilización de precipitación artificial (i.e., $25 \mathrm{~mm}$ en otoño, invierno y primavera) por parte de las plantas se estimó por la composición de isótopos de hidrógeno $\left(\delta^{2} \mathrm{H}\right)$ en el agua del xilema y los potenciales hídricos de pre-alba $\left(\psi_{\mathrm{pa}}\right)$ antes y después del riego. Los resultados indican que las tres especies utilizan una mezcla de dos fuentes de agua (agua superficial proveniente de las precipitaciones, y en mayor proporción, agua subterránea). A excepción de invierno, sólo las especies de sistemas radiculares dimórficos son capaces de reducir su déficit hídrico después de la aplicación de la precipitación artificial. La reducción en las precipitaciones observada en los últimos 100 años afectaría diferencialmente la productividad de las especies con sistema radicular dimórfico, y en especial al arbusto forrajero Balbisia peduncularis.
\end{abstract}

Palabras clave: fuentes de agua, proporción de isótopos estables, $\delta^{2} \mathrm{H}$, sistema radicular, desertificación, zonas áridas, Chile.

\begin{abstract}
Seasonal ability to use precipitation by three shrub species with different root systems (dimorphic: Balbisia peduncularis and Senna cummingii; deep: Haplopappus parvifolius) was evaluated in Quebrada El Romeral, north-central Chile. The site has an arid Mediterranean climate with coastal fog influence and an annual mean precipitation of $78 \mathrm{~mm}$ during the last 30 years. The use of artificial rain (i.e., $25 \mathrm{~mm}$ in fall, winter and spring) by plants was estimated by the hydrogen isotope composition $\left(\delta^{2} \mathrm{H}\right)$ in the xylem water and the pre-dawn water potentials $\left(\psi_{\mathrm{pa}}\right)$ before and after watering. The results show that the three species use a mixture of two water sources (shallow water coming from rainfall and a greater proportion of subterranean water). Except in winter, the species with dimorphic roots are able to reduce their water deficits after the artificial watering. The decrease in rainfall observed for the last 100 years would affect the productivity of the species with dimorphic root systems, especially the forage shrub Balbisia peduncularis.
\end{abstract}

Key words: water sources, stable isotopes ratio, $\delta^{2} \mathrm{H}$, root system, desertification, arid zone, Chile.

\section{INTRODUCCIÓN}

El crecimiento de las plantas que habitan en las zonas áridas tiene como principal limitante la escasez de agua (Noy-Meyer 1973, Gutiérrez 1993, 2001). Esta baja disponibilidad de agua afecta directamente las características del intercambio de gases, la productividad, la interacción entre especies y la estructura de la comunidad
(Hadley \& Szarek 1981, Ehleringer \& Mooney 1983, Arroyo et al. 1988, Rada et al. 1999, Squeo et al. 1999, 2000). Por ello, las especies vegetales de ecosistemas áridos optimizan la utilización de las fuentes de agua desarrollando diferentes estrategias para su adquisición, las que estarían relacionadas directamente con las formas de sus sistemas radiculares. Ehleringer et al. (1991) muestran que mientras los arbustos leñosos con 
raíces profundas presentan baja habilidad para utilizar agua desde las capas superficiales del suelo, muchas plantas herbáceas que poseen sistema radicular superficial pueden usar esta fuente de humedad para su crecimiento y reproducción.

Para las plantas de regiones áridas es beneficioso poseer raíces superficiales, las que les permitirían aprovechar las precipitaciones y/o el agua aportada por las neblinas y serían responsables de la adquisición de nutrientes (Cody 1986, Caldwell et al. 1990, 1998, Lynch 1995, Steudle 2000, 2001). Es conocido, que plantas con sistemas radiculares profundos son capaces de explotar fuentes de agua subterránea durante los períodos de sequía. Entre éstas últimas, las especies con sistemas radiculares dimórficos poseen raíces laterales que exploran las capas superficiales ricas en nutrientes durante la estación húmeda y la raíz principal es la que obtiene agua subterránea durante los períodos secos (Ehleringer et al. 1991, Caldwell et al. 1998).

La habilidad para cambiar rápidamente entre diferentes fuentes de agua representa una ventaja en la competencia por el agua dentro de los ecosistemas. En un estudio de la utilización de la humedad del suelo por el pino blanco del este (Pinus strobus) en el Este de Estados Unidos, White et al. (1985) mostraron que la extracción de agua cambia entre las capas superficiales y profundas del suelo, lo que dependería de la historia reciente de los eventos de precipitación. Inmediatamente después del evento de precipitación, los árboles de pino blanco utilizan la humedad desde las capas superficiales del suelo, presentando marcas isotópicas en el agua del xilema similares a las del reciente evento de precipitación. Sin embargo, cuando las capas superficiales del suelo comienzan a secarse, las marcas isotópicas del agua del xilema comienzan a decrecer progresivamente, indicando un cambio en la utilización de la humedad de la capa superficial a la humedad de las capas profundas del suelo.

Ehleringer et al. (1991) mostraron que las especies perennes (herbáceas, leñosas y suculentas) utilizaban el agua caída durante el invierno en un desierto frío al sur del estado de Utah. Sin embargo, durante el verano, sólo las especies anuales y suculentas de la comunidad utilizaron exclusivamente las precipitaciones de verano. Las especies perennes herbáceas y leñosas usaron diferentes mezclas de aguas caídas en verano e invierno (las perennes herbáceas usaron un $91 \%$ de las lluvias de verano y las leñosas un $57 \%$ ). En base a estos resultados, Ehleringer et al. (1991) postularon que los cambios en la precipitación de verano, predichos por los modelos de cambio climático global, resultarían en un incremento paulatino en la frecuencia de las especies perennes herbáceas y suculentas, mientras que la frecuencia de las especies leñosas podría disminuir.

Existen varios trabajos que han documentado cambios estacionales de las fuentes de agua que utilizan especies leñosas (i.e., agua del suelo superficial proveniente de las lluvias de verano y agua subterránea), mientras que otras especies mantienen invariante su fuente de agua, obteniéndola desde niveles más profundos del suelo durante todo el año (Ehleringer \& Cook 1991, Flanagan \& Ehleringer 1991, Flanagan et al. 1992, Valentini et al. 1992, Williams \& Ehleringer 2000, Schwinning \& Ehleringer 2001, Schwinning et al. 2002). Gregg (1991) entregó evidencias de un clino en la capacidad de respuesta a las lluvias de verano en una especie arbórea a lo largo de un gradiente geográfico, donde la fracción de precipitación de verano varía entre el 18 y el $40 \%$. Además, observó que los árboles de Juniperus osteosperma responden a la lluvia de verano sólo en los sitios de considerable precipitación de verano. No es sorprendente que exista una variación ecotípica en la estructura de la raíz a lo largo de un gradiente geográfico, pero es poco usual la falta de una respuesta inducida por las lluvias de verano en esos árboles de zonas áridas (Gibson \& Nobel 1986, Williams \& Ehleringer 2000).

En el desierto costero de la Cuarta Región de Coquimbo, co-habitan especies de arbustos con distinto sistema radicular, los que serían capaces de utilizar distintas fuentes de agua (Olivares \& Squeo 1999, Squeo et al. 1999, 2000).

El objetivo de este trabajo es evaluar la capacidad estacional de utilizar eventos de precipitación de tres especies de arbustos nativos con diferentes sistemas radiculares. Se plantea como hipótesis la existencia de una relación entre la distribución radicular de las especies y su estrategia de adquisición del recurso agua. Si esta hipótesis es correcta se podría predecir que las especies con sistemas radiculares dimórficos tendrían la capacidad de absorber el agua existente en las capas superficiales del suelo en los períodos húmedos, mientras que arbustos con sistema radicular profundo, utilizarían en forma exclusiva agua subterránea.

\section{MATERIALES Y MÉTODOS}

\section{Sitio de estudio}

El estudio fue realizado en la Quebrada Romeral $\left(29^{\circ} 43^{\prime} \mathrm{S}, 71^{\circ} 15^{\prime} \mathrm{O}, 300 \mathrm{~m}\right.$ de altitud), Cuarta Región de Coquimbo, norte-centro de Chile (Fig. 1). El lugar presenta un clima del tipo mediterráneo árido con influencia de neblinas. El promedio 
anual de precipitaciones de los últimos 30 años es $78 \mathrm{~mm}$ (Squeo et al. 1999). Durante 1998 se registró sólo un evento de precipitación ( 14 junio, $22,7 \mathrm{~mm}$ ). Las temperaturas medias mensuales oscilan entre 13 y $20^{\circ} \mathrm{C}$ y la humedad relativa promedio mensual varía entre 60 y $70 \%$.

La formación vegetal del sector corresponde a un Matorral Estepario Costero, el cual se encuentra formado por arbustos bajos de hojas duras (Gajardo 1994). La cobertura vegetal de especies arbustivas varía entre un 20 y $30 \%$ (Squeo et al. 1999). Las especies dominantes en los sectores de quebrada son Pleocarphus revolutus y Senna cumingii, mientras en la planicie la especie domi- nante es Haplopappus parvifolius, seguida de $S$. cumingii y $P$. revolutus (Olivares \& Squeo 1999).

\section{Especies estudiadas}

Se seleccionaron tres especies arbustivas características del desierto costero del norte-centro de Chile, las que según la clasificación de Squeo et al. (1999) - que se basa en los sistemas radiculares (superficial, dimórfico y/o profundo) y hábito (perennifolio, caducifolio) - pertenecerían a los siguientes grupos funcionales: (a) Balbisia peduncularis: perteneciente al grupo funcional

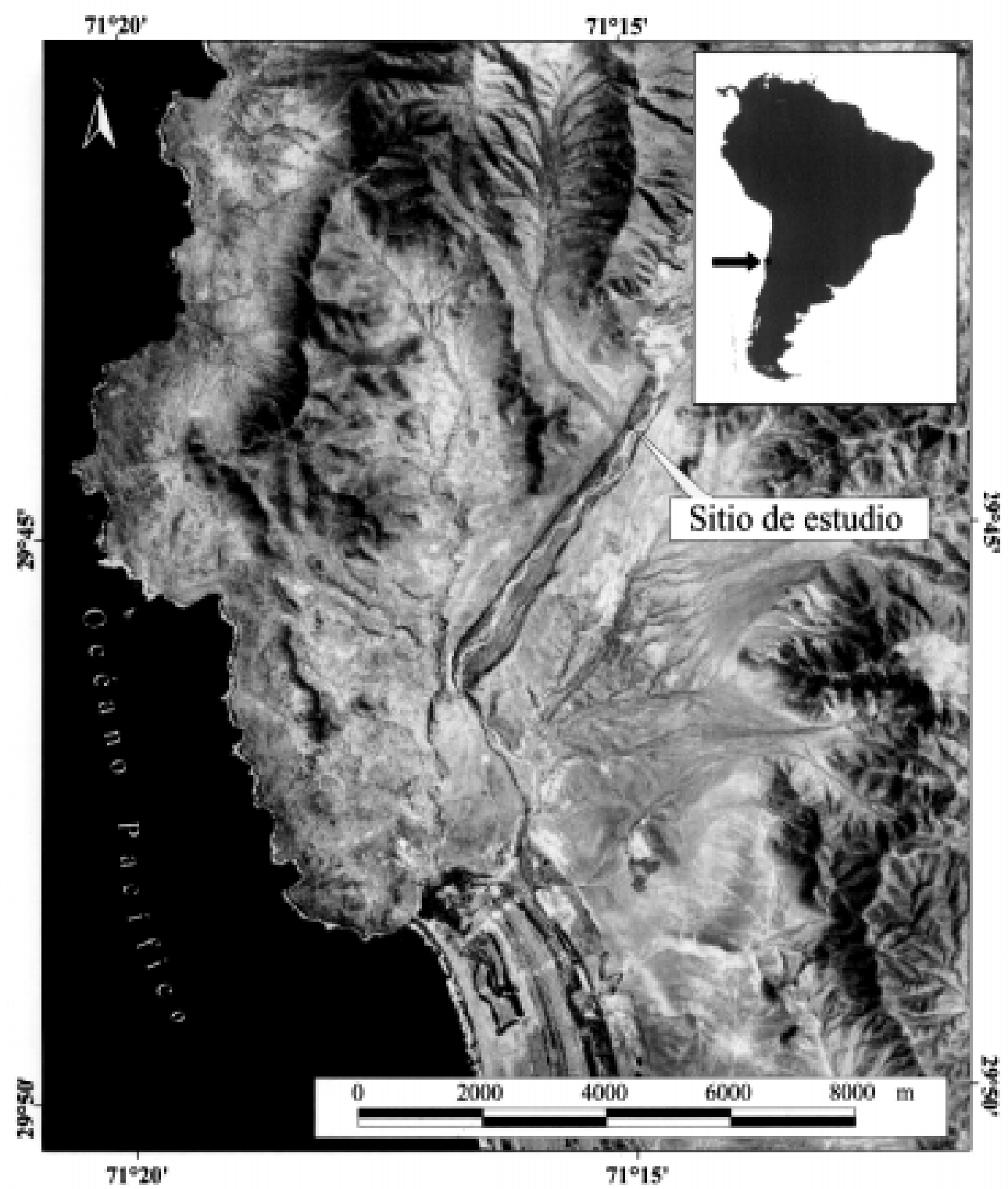

Fig. 1: Localización del sitio de estudio, Quebrada El Romeral, norte-centro de Chile. Location of the study site, Quebrada El Romeral, north-central Chile. 
TABLA 1

Contenido gravimétrico de agua del suelo (\% p/p) determinado en calicatas controles y regadas excavadas mayo, julio y octubre de 1998 en la Quebrada El Romeral

Soil water content (\% w/w) of control and irrigated samples collected in May, July and October 1998 in Quebrada El Romeral at two depths

\begin{tabular}{lcccccc}
\hline \multirow{2}{*}{ Profundidad } & \multicolumn{2}{c}{ Otoño } & \multicolumn{2}{c}{ Invierno } & \multicolumn{2}{c}{ Primavera } \\
Control & Regada & Control & Regada & Control & Regada \\
\hline $10-30 \mathrm{~cm}$ & $0,78 \pm 0,1$ & $7,64 \pm 0,1$ & $8,08 \pm 0,15$ & $9,30 \pm 0,11$ & $2,37 \pm 0,08$ & $7,04 \pm 0,11$ \\
$30-70 \mathrm{~cm}$ & $1,14 \pm 0,12$ & $2,21 \pm 0,1$ & $1,36 \pm 0,09$ & $2,57 \pm 0,13$ & $1,52 \pm 0,09$ & $1,87 \pm 0,12$ \\
\hline
\end{tabular}

de los arbustos caducifolios con sistema radicular dimórfico y/o profundo, que utilizan fuentes de agua superficiales y profundas de acuerdo a su disponibilidad. Esta especie presenta interés forrajero; (b) Senna cumingii: del grupo funcional de arbustos perennifolios con sistema radicular dimórfico capaces de utilizar ambas fuentes de agua, y potencialmente realizar levantamiento hidráulico; (c) Haplopappus parvifolius: representa el grupo funcional de arbustos perennifolios con sistema radicular profundo capaces de utilizar agua profunda.

Las tres especies estudiadas difieren en el cuociente entre la biomasa radicular y aérea de la planta (R/T) (Olivares et al. 19981). Balbisia peduncularis posee una mayor proporción de biomasa radicular $(\mathrm{R} / \mathrm{T}=1,0 \pm 0,2)$ en comparación con $S$. cumingii $(\mathrm{R} / \mathrm{T}=0,5 \pm 0,2)$, teniendo ambas especies un sistema radicular dimórfico. Esto sugiere que B. peduncularis eventualmente se podría comportar como una especie con sistema radicular más superficial dentro de su dimorfismo. La relación R/T de $H$. parvifolius es de 0,1 $\pm 0,05$.

\section{Diseño experimental}

El experimento se realizó en una exclusión de aproximadamente $1.500 \mathrm{~m}^{2}$ de superficie, cercada en su perímetro para evitar alteraciones provocadas por la entrada de ganado. El experimento consistió en simular un evento de precipitación

\footnotetext{
${ }^{1}$ OLIVARES N, CB JORQUERA, E GROTE, JR EHLERINGER \& FA SQUEO (1998) Arquitectura radicular y fuentes de agua utilizadas por especies arbustivas del desierto costero del norte-centro de Chile. Séptima Reunión Anual de la Sociedad de Ecología de Chile. La Serena, julio 24-25, 1998. Programa y Resúmenes: 10-11.
}

en tres estaciones del año (otoño, invierno y primavera). En cada estación del año se utilizaron 10 individuos por especie, seleccionando completamente al azar cinco réplicas para la aplicación del tratamiento (lluvia artificial) y otras cinco permanecieron como controles. Por lo tanto, en el experimento se utilizaron un total de 60 plantas, de las cuales 15 correspondieron a controles (cinco plantas $\mathrm{x}$ tres especies) y 45 a tratamientos (cinco plantas $\mathrm{x}$ tres especies $\mathrm{x}$ tres estaciones).

El riego se aplicó en los meses de mayo, julio y octubre de 1998, a través de una aspersión nocturna equivalente a una lluvia de $25 \mathrm{~mm}$ y de $3 \mathrm{~h}$ de duración, distribuida sobre parcelas circulares de $2 \mathrm{~m}^{2}$ de área con la planta ubicada en su centro.

\section{Descripción y análisis del suelo}

Se realizó una descripción del perfil del suelo a través de la excavación de una calicata de $2 \mathrm{~m}$ de profundidad. Desde los horizontes identificados se extrajeron muestras de suelo para la determinación del contenido gravimétrico de agua $\left(\mathrm{CGA}_{1}\right)$ (comparando peso fresco y peso seco, $105^{\circ} \mathrm{C}$ por 24 h) y de curvas de retención de humedad (CRH). La tensión de la humedad del suelo fue determinada mediante la técnica de "olla a presión" (Eejkelkemp Ag., Holanda), obteniéndose los contenidos gravimétricos de agua $\left(\mathrm{CGA}_{2}\right)$ presentes en el suelo a cuatro diferentes tensiones $(-0,03,-0,05,-0,5$ y $-1,5 \mathrm{MPa})$. A partir de las CRH se obtuvieron ecuaciones de regresión logarítmica, las cuales en conjunto con los $\mathrm{CGA}_{1}$ permitieron estimar los potenciales mátricos del suelo.

Determinación de la proporción de isótopos de hidrógeno $\left(\delta^{2} H\right)$

Para identificar las fuentes de agua utilizadas por cada especie se tomaron muestras de agua desde el estanque de riego, del pozo subterráneo Que- 
brada Romeral cercano a la parcela experimental y de un colector de neblina instalado en el sitio de estudio. También se colectaron tallos vegetales suberizados y muestras de suelo desde parcelas regadas y controles 2 días después de la aplicación de la lluvia artificial.

El agua del xilema y el agua contenida en las muestras de suelo fueron extraídas por medio de destilación criogénica al vacío (Ehleringer \& Osmond 1989, Dawson \& Ehleringer 1991). La determinación isotópica de $\delta^{2} \mathrm{H}$ se realizó a través del método de conversión del hidrógeno en gas (Coleman et al. 1982, modificado usando zinc de la Universidad de Indiana y a $500^{\circ} \mathrm{C}$ ). La composición isotópica de hidrógeno es expresada en notación delta $(\delta)$ y en partes por mil $(\% \circ)$ como:

$\delta=\left(\mathrm{R}_{\text {muestra }} / \mathrm{R}_{\text {estándar }}-1\right) \times 1.000 \%$,

donde $\mathrm{R}_{\text {muestra }} \mathrm{y} \mathrm{R}_{\text {estándar }}$ son las proporciones molares de deuterio/hidrógeno $\left({ }^{2} \mathrm{H} /{ }^{1} \mathrm{H}\right)$ de la muestra y estándar, respectivamente. El estándar es definido como SMOW (Standard Mean Ocean Water) con proporción absoluta de ${ }^{2} \mathrm{H} /{ }^{1} \mathrm{H}$ igual a 0,00015576 (Hagermann et al. 1970).

Para estimar el porcentaje de agua de lluvia tomada por las plantas en cada estación se utilizaron las marcas isotópicas de $\delta^{2} \mathrm{H}$ de las plantas control, plantas regadas y agua de lluvia. El porcentaje se calculó como el cuociente entre las diferencias isotópicas de plantas controles y regadas y de plantas controles y la marca isotópica del agua de lluvia (Lin et al. 1996): agua lluvia utilizada $(\%)=\left(\delta^{2} \mathrm{H}\right.$ controles $-\delta^{2} \mathrm{H}$ regadas $) /$ $\left(\delta^{2} \mathrm{H}\right.$ controles $-\delta^{2} \mathrm{H}$ lluvia artificial)

En el caso de las plantas controles se utilizó como marca isotópica el promedio de las cinco réplicas de la especie, mientras en las plantas regadas se utilizó la marca individual de cada réplica y para el agua de lluvia se usó la marca promedio ponderada del agua extraída desde los primeros $30 \mathrm{~cm}$ del suelo en cada estación.

\section{Medición de potenciales hídricos}

Para determinar la capacidad de utilizar agua desde las capas superficiales del suelo se midieron los potenciales xilemáticos de pre-alba en tres ocasiones durante cada estación: 1 día antes del riego, 2 días después del riego y 15 días después del riego. La técnica consistió en recolectar ramillas terminales activas desde cada una de las plantas en estudio (30 plantas por estación), utilizando para la medición una bomba de presión tipo Scholander (PMS Instrument Co., USA).

\section{Análisis estadístico}

Los resultados obtenidos fueron sometidos a un análisis de varianza (ANDEVA) para determinar la existencia de diferencias significativas entre especies, tratamientos, estaciones y entre sus interacciones. Para identificar qué variables presentan las diferencias se aplicó una prueba a posteriori de Tukey.

\section{RESULTADOS}

\section{Descripción y análisis de suelo}

El suelo analizado corresponde a una formación aluvial estratificada de acuerdo al tamaño de sus partículas, sin presencia de capas impermeables que limiten el movimiento del agua en el perfil y con un alto nivel de pedregosidad bajo el metro de profundidad. El perfil del suelo se divide en tres horizontes $(0-20,20-40$ y $40-200 \mathrm{~cm})$ de texturas predominantemente arenosas y de estructura en bloques subangulares altamente inestables. En el suelo no regado se identificó un sector superficial $(0-20 \mathrm{~cm})$ seco $(C G A<1 \%)$ y uno profundo $(170-$ $200 \mathrm{~cm})$ más húmedo $(\mathrm{CGA}=4,5$ a 9,0\%). En el suelo regado los CGA indican que el agua aplicada se distribuyó en los primeros $30 \mathrm{~cm}$ del suelo, aunque en algunos casos se detecta hasta los 50 $\mathrm{cm}$.

Las curvas de retención de humedad reflejan una escasa capacidad del suelo de retener humedad aprovechable por las raíces, capacidad que es levemente superior en el horizonte más superficial (Fig. 2).

\section{Potenciales hídricos}

Los potenciales mátricos del suelo $\left(\psi_{\mathrm{M}}\right)$ fueron comparados con los potenciales hídricos $\left(\psi_{\mathrm{H}}\right)$ medidos en las plantas dos días después del riego, infiriéndose por similitud de valores la profundidad desde donde las plantas extrajeron agua en cada estación (Fig. 3): (a) Otoño: los controles de las tres especies y las plantas regadas de Haplopappus parvifolius estarían extrayendo agua desde el intervalo de profundidad $80-90 \mathrm{~cm}$, mientras las plantas regadas de Balbisia peduncularis y Senna cumingii lo harían desde un estrato más superficial (40-50 cm) (Fig. 3A); (b) Invierno: todas las plantas de $B$. peduncularis y $S$. cumingi extraen agua desde el intervalo de profundidad 20-30 cm, mientras las de $H$. parvifolius lo hacen desde un estrato más profundo $(40-60 \mathrm{~cm})($ Fig. 3B); (c) Primavera: las plantas regadas de $B$. 


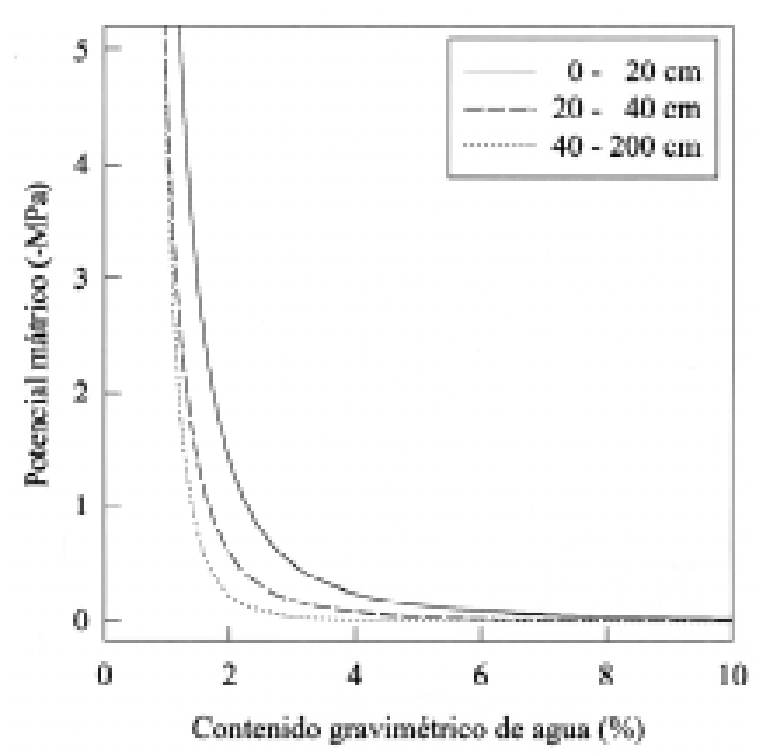

Fig. 2: Curvas de retención de humedad obtenidas a partir de muestras de suelo extraídas de los horizontes identificados en la calicata de $2 \mathrm{~m}$ de profundidad excavada en mayo de 1998 en la Quebrada El Romeral. Las ecuaciones de regresión entre el potencial mátrico del suelo $\left(\psi_{\mathrm{m}}\right)$ y el contenido gravimético de agua (cga) para cada horizonte son: $0-20 \mathrm{~cm}: \psi_{\mathrm{m}}=\mathrm{e}^{2,089-2,584 \operatorname{Ln} \text { cga }}, 20-40$ $\mathrm{cm}: \psi_{\mathrm{m}}=\mathrm{e}^{1,475-2,922 \text { Ln cga }}, 40-200 \mathrm{~cm}: \psi_{\mathrm{m}}=\mathrm{e}^{1,382-4,217}$ Ln cga.

Water retention curves obtained from soil samples collected at the horizons identified in the test pit of $2 \mathrm{~m}$ excavated in May 1988 in Quebrada El Romeral. The regression equations between the soil matric potential $\left(\psi_{\mathrm{m}}\right)$ and the gravimetric water content (cga) for each horizon are: $0-20 \mathrm{~cm}: \psi_{\mathrm{m}}=\mathrm{e}^{2.089-2.584 \mathrm{Ln} \mathrm{cga}}, 20-40 \mathrm{~cm}: \psi_{\mathrm{m}}=\mathrm{e}^{1.475-2.922}$ Ln cga $, 40-200 \mathrm{~cm}: \psi_{\mathrm{m}}=\mathrm{e}^{1.382-4.217 \text { Ln cga }}$. peduncularis y $S$. cumingii extraen agua desde el intervalo de profundidad 60-70 cm (Fig. 3C).

Proporción de isótopos estables de hidrógeno $\left(\delta^{2} H\right)$

Las fuentes de agua registran diferentes composiciones isotópicas que permiten diferenciarlas claramente (Tabla 2). El agua extraída del suelo regado y sin riego presenta diferencias isotópicas en los primeros $30 \mathrm{~cm}$ de profundidad con marcas empobrecidas en deuterio en el suelo regado (Tabla 3).

En las tres estaciones analizadas, las tres especies disminuyen sus valores de $\delta^{2} \mathrm{H}$ después del riego indicando que ha ocurrido absorción del agua agregada (Fig. 4). Las proporciones de isótopos de hidrógeno $\left(\delta^{2} \mathrm{H}\right)$ en el agua del xilema presentan diferencias significativas entre estaciones, especies, tratamientos y estación $\mathrm{x}$ tratamiento $(\mathrm{P}<0,001)$. Los análisis a posteriori indican que invierno, a nivel de estación, y $B$. peduncularis, a nivel de especie, presentan los mayores valores de $\delta^{2} \mathrm{H}$ (Tabla 4). Las plantas regadas presentan valores de $\delta^{2} \mathrm{H}$ menores (-61,6 $\pm 2,3 \%$ ) que las controles $(-49,9 \pm 1,86 \%$ ) indicando que efectivamente el agua agregada fue absorbida por las plantas. Las marcas empobrecidas en deuterio exhibidas por las plantas regadas se mantienen en las tres estaciones, presentándose las mayores diferencias entre tratamientos en primavera (Tabla 4).

Los porcentajes de agua de lluvia artificial utilizada por las plantas presentan diferencias significativas sólo entre estaciones $(\mathrm{P}=0,01)$, mostrando los análisis a posteriori que la proporción de lluvia artificial utilizada en invierno ( $17 \pm 4,8$ $\%)$ y primavera $(22 \pm 3,2 \%)$ es significativamente mayor que la empleada en otoño $(7 \pm 3,5 \%)$. A

TABLA 2

Proporción de isótopos de hidrógeno $\left(\delta^{2} \mathrm{H}, \%\right.$ ) de las potenciales fuentes de agua, determinadas en muestras colectadas en otoño (mayo), invierno (julio) y primavera (octubre) de 1998 en la Quebrada El Romeral

Hydrogen isotope ratios $\left(\delta^{2} \mathrm{H}, \%\right.$ of of the potential water sources in samples collected in fall (May), winter (July) and spring (October) of 1998 in Quebrada El Romeral

\begin{tabular}{|c|c|c|c|c|}
\hline Fuente de agua & Otoño & Invierno & Primavera & Promedio \\
\hline Lluvia artificial & $-101,0$ & $-100,0$ & $-100,0$ & $-100,7 \pm 2,0$ \\
\hline Agua subterránea & $-43,0$ & $-41,0$ & $-36,0$ & $-40,0 \pm 2,1$ \\
\hline Lluvia natural* & & $-21,0$ & & $-21,0$ \\
\hline
\end{tabular}

*Lluvia natural corresponde a la precipitación única ocurrida el 14 de junio de 1998 en el sitio de estudio (22,7 mm), un mes antes del experimento de invierno 
pesar de que no se encontraron diferencias significativas entre especies $(\mathrm{P}=0,14)$ existe una tendencia que muestra que $B$. peduncularis y $H$. parvifolius utilizarían los porcentajes más altos

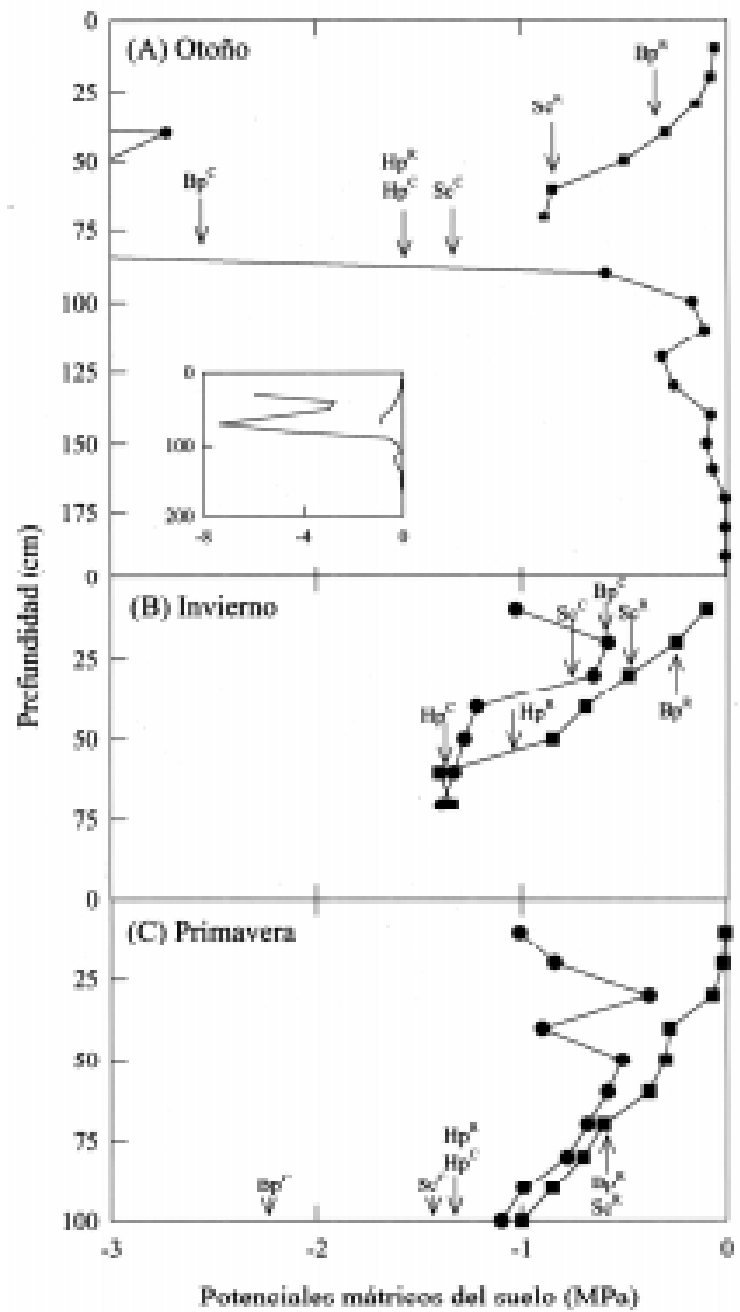

Fig. 3: Potenciales mátricos del suelo regado (cuadrado) y sin riego (círculo), determinados en: (A) otoño (mayo de 1998), (B) invierno (julio) y (C) primavera (octubre) en la Quebrada El Romeral. Las flechas indican el potencial xilemático de pre-alba de las especies estudiadas; $\mathrm{Bp}=$ Balbisia peduncularis, $\mathrm{Hp}=$ Haplopappus parvifolius, $\mathrm{Sc}=$ Senna cumingii $; \mathrm{R}=$ regada, $\mathrm{C}=$ control. El inserto en Otoño muestra los datos completos del potencial mátrico del suelo según profundidad.

Soil matric potentials for control (circle) and irrigated soils (square) determined in: (A) fall (May 1998), (B) winter (July) and (C) spring (October) in Quebrada El Romeral. The arrows indicate the pre-dawn xylematic potential of the studied species; $\mathrm{Bp}=$ Balbisia peduncularis, $\mathrm{Hp}=$ Haplopappus parvifolius, $\mathrm{Sc}=$ Senna cumingii $; \mathrm{R}=$ watered, $\mathrm{C}=$ control. The insert in fall shows the entire data of soil matric potentials at different depths.

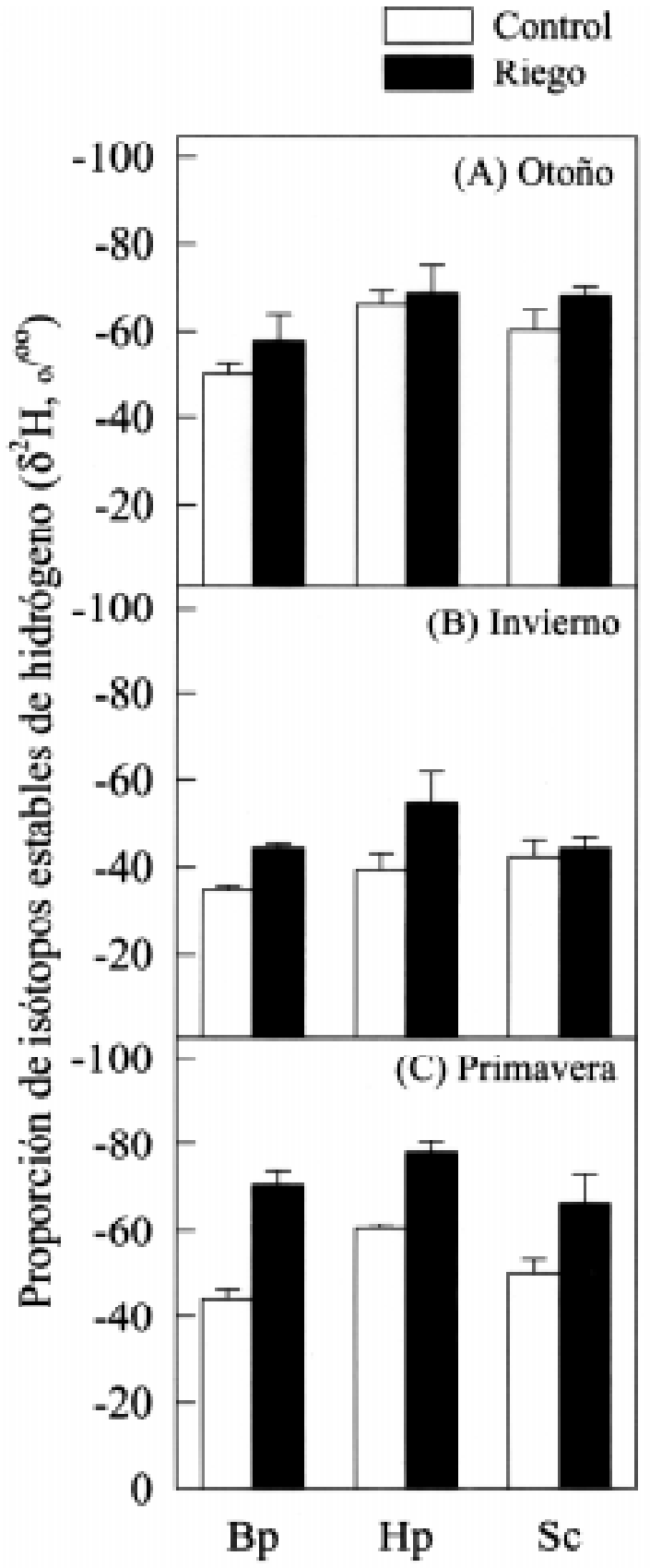

Fig. 4: Proporción de isótopos estables de hidrógeno $\left(\delta^{2} \mathrm{H}, \%\right.$ o en agua extraída de xilema en plantas control y regadas en: (A) otoño (mayo de 1998), (B) invierno (julio) y (C) primavera (octubre) en la Quebrada El Romeral. Se indica el promedio y un error estándar; $\mathrm{Bp}=$ Balbisia peduncularis, $\mathrm{Hp}=$ Haplopappus parvifolius, $\mathrm{Sc}=$ Senna cumingii.

Hydrogen isotope ratios $\left(\delta^{2} \mathrm{H}, \%\right.$ ) from xylem water of control and watered plants in: (A) fall (May 1998), (B) winter (July) and (C) spring (October) in Quebrada El Romeral. Means and one standard error are shown; $\mathrm{Bp}=$ Balbisia peduncularis, $\mathrm{Hp}=$ Haplopappus parvifolius, $\mathrm{Sc}=$ Senna cumingii. 
TABLA 3

Proporción de isótopos de hidrógeno $\left(\delta^{2} \mathrm{H}, \%\right.$ ) en el agua presente en el suelo, determinadas en muestras obtenidas en las calicatas excavadas otoño (mayo), invierno (julio) y primavera

(octubre) de 1998 en la Quebrada El Romeral

Hydrogen isotope ratios $\left(\delta^{2} \mathrm{H}, \%\right.$ o of the soil water samples collected in fall (May), winter (July) and spring (October) of 1998 in Quebrada El Romeral at different depths

\begin{tabular}{lcccccc}
\hline Profundidad & $\begin{array}{c}\text { Otoño } \\
\text { Control }\end{array}$ & $\begin{array}{c}\text { Invierno } \\
\text { Regada }\end{array}$ & $\begin{array}{c}\text { Primavera } \\
\text { Control }\end{array}$ & Regada & Control & Regada \\
\hline $0-10 \mathrm{~cm}$ & -41 & -88 & -9 & -88 & -40 & -110 \\
$10-20 \mathrm{~cm}$ & -56 & -95 & -19 & -56 & -34 & -90 \\
$20-30 \mathrm{~cm}$ & -53 & -87 & -39 & -34 & -38 & -59 \\
$30-40 \mathrm{~cm}$ & -52 & -51 & -35 & -16 & -52 & -46 \\
$40-50 \mathrm{~cm}$ & -30 & -55 & -33 & -32 & -47 & -59 \\
\hline
\end{tabular}

de lluvia artificial, derivando el $18 \%$ del agua presente en sus xilemas desde el agua de riego, mientras en $S$. cumingii este porcentaje alcanzaría sólo al $10 \%$.

\section{Potenciales hídricos de pre-alba $\left(\psi_{p a}\right)$}

Los potenciales hídricos de pre-alba (Tablas 5 y 6) presentan diferencias significativas en la medición: (a) un día antes del riego: entre estaciones, especies y en la interacción estación x especie $(P<0,001)$, pero no entre tratamientos (control-regadas); (b) dos días después del riego: entre estaciones, especies, tratamientos y en las interacciones estación $\mathrm{x}$ especie, tratamiento $\mathrm{x}$ especie y estación $\mathrm{x}$ tratamiento $\mathrm{x}$ especie $(\mathrm{P}<$ 0,001); y (c) quince días después del riego: entre estaciones y tratamientos $(\mathrm{P}<0,001)$.

Los análisis a posteriori muestran que invierno (a nivel de estación) y B. peduncularis (a nivel de especie) presentan los mayores $\psi_{\text {pa }}$ (Tabla 5). En el caso de los tratamientos, las plantas regadas son las que presentan los potenciales hídricos más cercanos a cero.

La interacción estación $\mathrm{x}$ especie muestra que B. peduncularis presenta diferencias estacionales caracterizadas por $\psi_{\mathrm{pa}}$ en invierno significativamente superiores a los de otoño y primavera antes y después del riego (Tabla 5). En la medi-

TABLA 4

Proporción de isótopos de hidrógeno $\left(\delta^{2} \mathrm{H}, \%\right.$ ) del agua extraída del xilema en otoño (mayo), invierno (julio) y primavera (octubre) de 1998 en la Quebrada El Romeral. Cada valor representa la media y el error estándar. Diferencias estadísticas entre las medias para las comparaciones Especies, Estación, Tratamiento y la interacción Tratamiento x Estación son representadas con distintas letras

Hydrogen isotope ratios $\left(\mathrm{d}^{2} \mathrm{H}, \%\right.$ ) of xylem water for fall (May), winter (July) and spring (October) of 1998 in Quebrada El Romeral. Each value correspond to the mean and one standard error. Statistical differences among means for species, season, treatment and treatment $\mathrm{x}$ season interaction are represented with different letters

\begin{tabular}{lcccc}
\hline (A) Especie versus estación & Otoño & Invierno & Primavera & Promedio especie \\
\hline B. peduncularis & $-53,80 \pm 3,76$ & $-39,55 \pm 1,88$ & $-59,11 \pm 4,81$ & $-50,50 \pm 2,54 \mathrm{a}$ \\
H. parvifolius & $-67,90 \pm 3,72$ & $-47,25 \pm 4,82$ & $-69,20 \pm 3,27$ & $-61,40 \pm 2,90 \mathrm{~b}$ \\
S. cumingii & $-64,45 \pm 3,13$ & $-43,33 \pm 2,42$ & $-58,40 \pm 4,74$ & $-55,40 \pm 2,58 \mathrm{c}$ \\
Promedio estacional & $-62,10 \pm 2,27^{\mathrm{a}}$ & $-43,40 \pm 1,93 \mathrm{~b}$ & $-62,30 \pm 2,57 \mathrm{a}$ & \\
\hline
\end{tabular}

(B) Tratamiento versus estación

\begin{tabular}{lcccc} 
& \multicolumn{1}{c}{ Otoño } & Invierno & Primavera & Promedio tratamiento \\
\hline Controles & $-58,90 \pm 2,91 \mathrm{a}$ & $-38,90 \pm 2,01 \mathrm{~b}$ & $-52,21 \pm 2,21 \mathrm{c}$ & $-49,90 \pm 1,86 \mathrm{a}$ \\
Regadas & $-65,20 \pm 3,38 \mathrm{~d}$ & $-47,80 \pm 2,92 \mathrm{c}$ & $-71,80 \pm 2,95 \mathrm{~d}$ & $-61,60 \pm 2,32 \mathrm{~b}$ \\
Promedio estacional & $-62,10 \pm 2,27 \mathrm{a}$ & $-43,40 \pm 1,93 \mathrm{~b}$ & $-62,30 \pm 2,57 \mathrm{a}$ & \\
\hline
\end{tabular}


TABLA 5

Potenciales hídricos de pre-alba (MPa) medidos en otoño (mayo), invierno (julio) y primavera (octubre) de 1998 en la Quebrada El Romeral. Cada valor representa la media y un error estándar. Diferencias estadísticas entre las medias para las comparaciones Especie, Estación y la interacción Especie x Estación son representadas con distintas letras

Pre-dawn water potential (MPa) for fall (May), winter (July) and spring (October) of 1998 in Quebrada El Romeral. Each value corresponds to the mean and one standard error. Statistical differences among means for species, season and species $x$ season interaction are represented with different letters

(A) Un día antes del riego

\begin{tabular}{lcccc} 
Especie/estación & Otoño & Invierno & Primavera & Promedio especie \\
\hline B. peduncularis & $-3,15 \pm 0,17 \mathrm{a}$ & $-0,52 \pm 0,03 \mathrm{~b}$ & $-3,04 \pm 0,21 \mathrm{a}$ & $-2,24 \pm 0,24 \mathrm{a}$ \\
H. parvifolius & $-1,43 \pm 0,11 \mathrm{~b}, \mathrm{c}$ & $-0,94 \pm 0,04 \mathrm{~b}$ & $-1,71 \pm 0,10 \mathrm{c}$ & $-1,36 \pm 0,08 \mathrm{~b}$ \\
S. cumingii & $-1,19 \pm 0,12 \mathrm{~b}, \mathrm{c}$ & $-0,54 \pm 0,04 \mathrm{~b}$ & $-2,15 \pm 0,15 \mathrm{c}$ & $-1,29 \pm 0,14 \mathrm{~b}$ \\
Promedio estación & $-1,92 \pm 0,18 \mathrm{a}$ & $-0,67 \pm 0,04 \mathrm{~b}$ & $-2,30 \pm 0,14 \mathrm{a}$ &
\end{tabular}

(B) Dos días después del riego

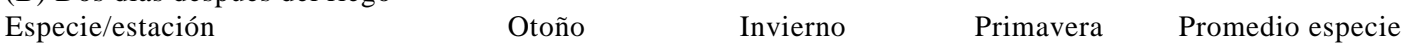

\begin{tabular}{lllll}
\hline B. peduncularis & $-2,24 \pm 0,16 \mathrm{a}$ & $-0,71 \pm 0,03 \mathrm{~b}$ & $-1,96 \pm 0,49 \mathrm{a}$ & $-1,64 \pm 0,21 \mathrm{a}$ \\
$H$. parvifolius & $-1,47 \pm 0,08 \mathrm{c}$ & $-1,05 \pm 0,04 \mathrm{~b}$ & $-1,29 \pm 0,08 \mathrm{c}$ & $-1,27 \pm 0,05 \mathrm{a}, \mathrm{b}$ \\
S. cumingii & $-1,13 \pm 0,15 \mathrm{c}$ & $-0,53 \pm 0,03 \mathrm{~b}$ & $-1,01 \pm 0,18 \mathrm{~b}, \mathrm{c}$ & $-0,89 \pm 0,09 \mathrm{~b}$ \\
Promedio estación & $-1,61 \pm 0,11 \mathrm{a}$ & $-0,76 \pm 0,04 \mathrm{~b}$ & $-1,42 \pm 0,19 \mathrm{a}$ &
\end{tabular}

(C) 15 días después del riego

\begin{tabular}{lcccc} 
Especie/estación & Otoño & Invierno & Primavera & Promedio especie \\
\hline B. peduncularis & $-1,19 \pm 0,32 \mathrm{a}$ & $-0,44 \pm 0,07 \mathrm{c}$ & $-2,03 \pm 0,56 \mathrm{~d}$ & $-1,22 \pm 0,24$ \\
H. parvifolius & $-1,38 \pm 0,05 \mathrm{a}$ & $-1,01 \pm 0,05 \mathrm{a}$ & $-1,21 \pm 0,10 \mathrm{a}$ & $-1,20 \pm 0,05$ \\
S. cumingii & $-0,86 \pm 0,09 \mathrm{~b}$ & $-0,56 \pm 0,06 \mathrm{c}$ & $-1,70 \pm 0,22 \mathrm{e}$ & $-1,04 \pm 0,12$ \\
Promedio estación & $-1,14 \pm 0,11 \mathrm{a}$ & $-0,67 \pm 0,06 \mathrm{~b}$ & $-1,64 \pm 0,20 \mathrm{c}$ & \\
\hline
\end{tabular}

ción anterior al riego, H. parvifolius y $S$. cumingii presentan $\psi_{\text {pa }}$ en invierno significativamente mayores a los de primavera (Tabla 5A), mientras en la medición realizada 2 días después del riego $S$. cumingii presenta $\psi_{\mathrm{pa}}$ en invierno mayores a los de otoño y $H$. parvifolius registra valores en invierno superiores a los de otoño y primavera (Tabla 5B). En ésta última especie, la diferencia estacional desaparece a los 15 días post-riego (Tabla 5C). Adicionalmente, para las mediciones realizadas 1 día ante y 2 días después del riego, la interacción especie $x$ estación indica que en invierno no existen diferencias significativas entre los $\psi_{\mathrm{pa}}$ de las tres especies, mientras en otoño y primavera $B$. peduncularis presenta $\psi_{\mathrm{pa}}$ significativamente menores (Tabla 5A y 5B).

La interacción tratamiento $\mathrm{x}$ especie muestra que los $\psi_{\text {pa }}$ de B. peduncularis y S. cumingii se incrementan significativamente después del riego, mientras los de $H$. parvifolius no sufren variaciones significativas (Tabla 6). Sin embargo, $B$. peduncularis y $S$. cumingii aumentan significativamente los $\psi_{\mathrm{pa}}$ después del riego sólo en primavera (Fig. 5).

\section{TABLA 6}

Potenciales hídricos de pre-alba (MPa) de plantas controles y regadas, medidos dos días después de cada riego en la Quebrada El Romeral. Cada valor representa la media y el error estándar. Diferencias estadísticas entre tratamientos son representadas con distintas letras

Pre-dawn water potential (MPa) of control and watered plants measured two days after watering in Quebrada El Romeral. Each value corresponds to the mean and one standard error. Statistical differences among treatment are represented with different letters

\begin{tabular}{lcc}
\hline Especie/tratamiento & Controles & Regadas \\
\hline B. peduncularis & $-2,20 \pm 0,31 \mathrm{a}$ & $-1,16 \pm 0,22 \mathrm{~b}$ \\
H. parvifolius & $-1,29 \pm 0,07 \mathrm{~b}$ & $-1,25 \pm 0,07 \mathrm{~b}$ \\
S. cumingii & $-1,09 \pm 0,15 \mathrm{a}$ & $-0,68 \pm 0,07 \mathrm{~b}$ \\
Promedio tratamiento & $-1,50 \pm 0,13 \mathrm{a}$ & $-1,03 \pm 0,09 \mathrm{~b}$ \\
\hline
\end{tabular}




\section{DISCUSIÓN}

\section{Capacidad de utilizar eventos de precipitación}

Estudios previos han utilizado la composición isotópica del agua del xilema para estimar la utilización de lluvias de verano y otras fuentes de agua, por especies leñosas desérticas (Aravena \&

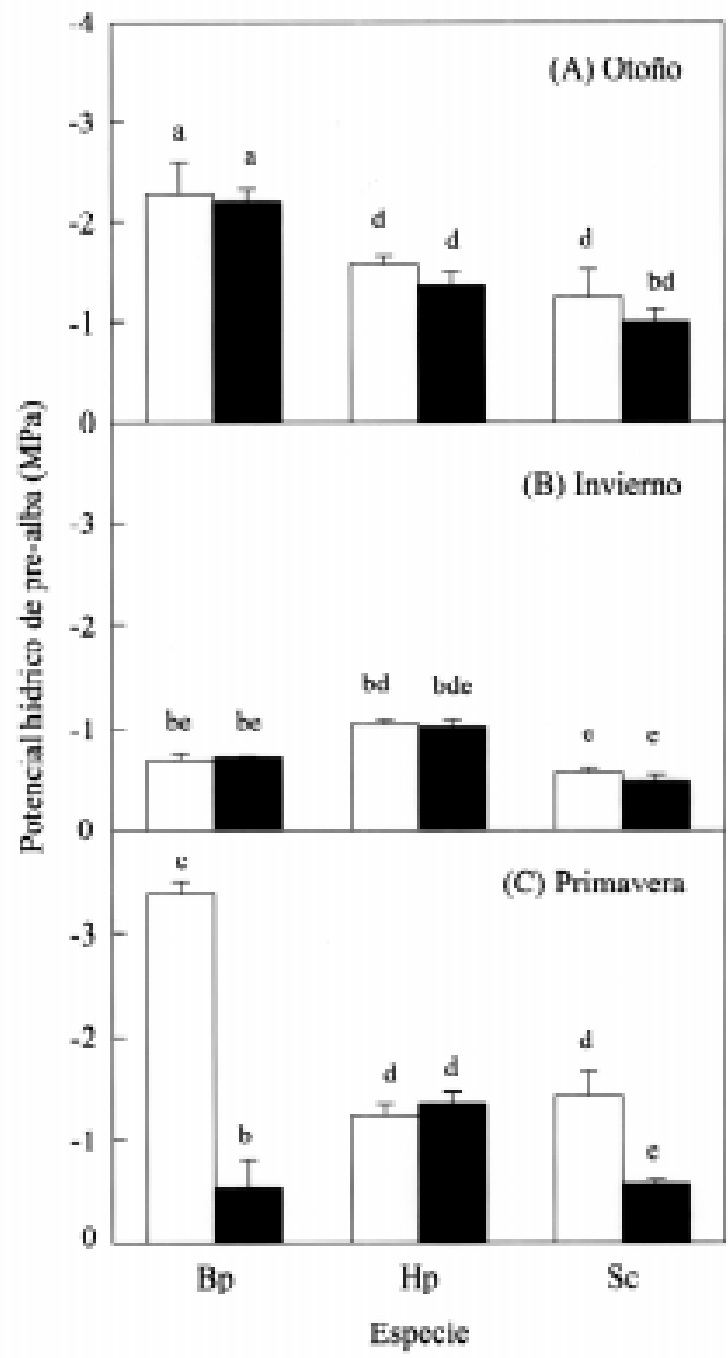

Fig. 5: Potenciales hídricos de pre-alba (MPa) de plantas controles y regadas en cada estación, medidos dos días después del riego en: (A) otoño (mayo de 1998), (B) invierno (julio) y (C) primavera (octubre) en la Quebrada El Romeral. Se indica el promedio y un error estándar; $\mathrm{Bp}=$ Balbisia peduncularis, $\mathrm{Hp}=$ Haplopappus parvifolius, $\mathrm{Sc}=$ Senna cumingii .

Pre-dawn water potential (MPa) of control and watered plants in each season, measured two days after watering in: (A) fall (May 1998), (B) winter (July) and (C) spring (October) in Quebrada El Romeral. Means and one standard error are shown; $\mathrm{Bp}=$ Balbisia peduncularis, $\mathrm{Hp}$ = Haplopappus parvifolius, $\mathrm{Sc}=$ Senna cumingii .
Acevedo 1985, Dawson \& Ehleringer 1991, Lin et al. 1996). En ecosistemas como el secano costero de la Cuarta Región de Coquimbo y específicamente en el sector Quebrada El Romeral, el empleo de esta técnica se facilita debido a que la proporción de isótopos estables de hidrógeno de las fuentes de agua naturales difieren entre si (agua subterránea $=-40 \%$ y agua de neblina $=$ $-12 \%$ ) y con la fuente de agua artificial que proviene del río Elqui $(-100 \%)$.

Los valores de $\delta^{2} \mathrm{H}$ registrados en el suelo que no fue regado (Tabla 3 ) y en el xilema de las plantas control (Tabla 4), se asemejan a la composición isotópica del agua subterránea (Tabla 2), lo que descarta una contribución importante del agua proveniente de neblinas y sugiere que en ausencia de precipitaciones, las especies se abastecen esencialmente de agua profunda. Los análisis de $\delta^{2} \mathrm{H}$ indican que la composición isotópica del agua de riego se detecta hasta $\operatorname{los} 30 \mathrm{~cm}$ de profundidad y que las plantas regadas de las tres especies presentan menores valores de $\delta^{2} \mathrm{H}$ dos días después del riego en comparación con las controles. Esto sugiere que sus raíces superficiales se encuentran funcionalmente activas, y que eventualmente serían capaces de aprovechar precipitaciones y ser responsables de la adquisición de nutrientes, tal como ha sido sugerido para otras especies (Ehleringer et al. 1991, Caldwell et al.1998, Olivares et al. 1998).

Los diferentes valores de $\delta^{2} \mathrm{H}$ registrados por plantas control y regadas representan sólo una pequeña fracción en la gran diferencia existente entre las marcas isotópicas del agua de riego y el agua subterránea. El escaso uso de agua de riego se refleja en los porcentajes de utilización de lluvia artificial, calculados a partir de la proporción isotópica, donde en el mejor de los casos Balbisia peduncularis y Haplopappus parvifolius derivan sólo el $18 \%$ del agua presente en su xilema desde la precipitación artificial, mientras en Senna cumingii este porcentaje apenas alcanza al $10 \%$. Basados en los análisis de isótopos estables de hidrógeno, se estima que las tres especies utilizarían una mezcla desigual de dos fuentes de agua la que estaría compuesta por agua superficial proveniente del riego en menor proporción y por agua subterránea en mayor proporción. La cantidad de agua superficial utilizada por las especies estudiadas en este trabajo son menores que las registradas por Lin et al. (1996) en el desierto de Colorado, en que después de la aplicación de una lluvia artificial Coleogyne ramossisima utilizó agua de lluvia en porcentajes superiores al $50 \%$.

Las especies, con su raíz principal profunda serían capaces de explotar fuentes de agua subte- 
rránea durante los períodos secos, mientras con sus raíces laterales serían capaces de explorar las capas ricas en nutrientes durante la estación húmeda (Ehleringer et al. 1991, Caldwell et al. 1998, Olivares et al. 1998). Estos resultados concuerdan con los obtenidos por Squeo et al. (1999) en 14 especies arbustivas en la Quebrada El Romeral, donde a través de análisis de $\delta^{2} \mathrm{H}$ determinaron el uso directo de dos fuentes de agua: las primeras asociadas a un estrato superficial no superior a $\operatorname{los} 50 \mathrm{~cm}$ de profundidad (zona del suelo recargada directamente por las precipitaciones) y otras a un estrato más profundo que se asocia a aguas subterráneas. La capacidad de las plantas de utilizar la humedad de las capas profundas del suelo las favorece en situaciones ecológicas naturales, dándoles ventaja sobre las especies que dependen exclusivamente de las capas superficiales, especialmente cuando están expuestas a déficit hídricos prolongados (Ehleringer \& Dawson 1992).

Adicionalmente, nuestros resultados muestran un importante efecto estacional caracterizado por un significativo incremento en los valores de $\delta^{2} \mathrm{H}$ registrados en las plantas en invierno. Este comportamiento se asoció directamente con la única precipitación natural $\left(22,7 \mathrm{~mm}, \delta^{2} \mathrm{H}=-21 \%\right.$ ) ocurrida a finales de junio de 1998 , por lo que se estimó que en invierno las especies utilizaron una mezcla de agua en la que participó la lluvia natural como un nuevo componente exclusivo de esta estación.

Los potenciales hídricos de pre-alba medidos antes y después del riego permiten estimar la capacidad de las especies de utilizar agua proveniente de precipitaciones. Esta capacidad se demuestra a través de cambios en el estado hídrico de las plantas, expresados como reducción del déficit hídrico. Este parámetro permitió distinguir al menos dos respuestas distintas a los eventos de precipitación artificial. La primera de $B$. peduncularis y $S$. cumingii que, con incrementos significativos en sus potenciales hídricos después del riego, demostraron la capacidad de aprovechar la mayor disponibilidad de agua superficial. Esta capacidad fue más notoria en primavera, lo que estaría relacionado directamente con la mayor actividad radicular exhibida por las especies en esta estación (Olivares \& Squeo 1999). En contraste, la ausencia de diferencias entre tratamientos en invierno se debería a la mayor humedad existente en el suelo superficial producto de la precipitación ocurrida a finales de otoño de 1998 (un mes antes del riego de invierno). Este evento natural explicaría la disminución en el déficit hídrico exhibido por las plantas controles en invierno, y por lo tanto, sería el causante de la similitud existente entre los potenciales hídricos de las plantas de ambos tratamientos en esta estación.

Balbisia peduncularis, además de registrar las mayores diferencias entre tratamientos, presentó potenciales hídricos significativamente menores que las otras especies en otoño y primavera, lo que se asocia directamente con la escasez de agua superficial existente en el suelo sin riego en estas estaciones. Adicionalmente, esta especie registró variaciones estacionales significativas caracterizadas por mayores potenciales hídricos en invierno cuando el contenido de humedad superficial fue mayor, y menores en otoño y primavera cuando el suelo superficial estuvo más seco. Este comportamiento sugiere la existencia de una estrecha relación entre el estado hídrico de la especie y el contenido de agua del suelo superficial. Por esta razón, se estima que $B$. peduncularis es la especie que mejor respondería a aumentos en la disponibilidad de agua superficial y que por lo tanto es la más dependiente de la ocurrencia de precipitaciones.

Una respuesta distinta mostró Haplopappus parvifolius, que aún cuando incorporó agua de riego $(18 \%)$ y presentó un $98 \%$ de su biomasa radicular acumulada en los primeros $60 \mathrm{~cm}$ de profundidad (Olivares et al. 1998), no mostró diferencias significativas en sus potenciales hídricos entre plantas controles y regadas después del riego, demostrando que no fue capaz de utilizar el agua superficial para disminuir su déficit hídrico y que, por lo tanto, sería indiferente a la ocurrencia de precipitaciones de magnitud moderada $(25 \mathrm{~mm})$. Un comportamiento parecido fue observado por Flanagan \& Ehleringer (1991) en Artemisia tridentata, donde detectaron que pese a que la mayoría de las raíces de la especie se localizan a menos de un metro de profundidad, sus raíces profundas (> $3 \mathrm{~m}$ ) fueron las responsables de la absorción de agua. Estos comportamientos confirman el hecho que la mayoría de la biomasa radicular se localice en las capas superiores del suelo no significa que todas esas raíces estén funcionalmente activas para absorber agua o que el agua es extraída de esa zona (Squeo et al. 1999).

Happlopapus parvifolius, pese a registrar aumentos en sus potenciales hídricos de invierno, presentó un comportamiento más bien homogéneo a través de las estaciones, reflejando nuevamente su baja dependencia de la humedad superficial del suelo. Sin embargo, en esta especie de sistema radicular profundo, se observó un comportamiento estacional caracterizado por potenciales hídricos significativamente más altos en invierno. La disminución del déficit hídrico exhi- 
bido por esta especie en invierno, se debería a que sus raíces superficiales serían capaces de utilizar agua superficial cuando el contenido de humedad del suelo bordea el $10 \%$ como sucedió a finales de otoño y principios de invierno cuando el suelo recibió con un mes distanciamiento dos precipitaciones superiores a los $20 \mathrm{~mm}$ cada una (natural y artificial). Estos resultados sugieren que $H$. parvifolius eventualmente sería capaz de utilizar agua superficial en las estaciones húmedas. Este comportamiento coincide con lo observado por Squeo et al. (1999) en la Quebrada Romeral, quienes determinaron que, en un año inusualmente lluvioso todas las especies, independientes de su distribución radicular, fueron capaces de utilizar directamente el agua de lluvia. Esta conducta ya ha sido descrita en especies leñosas de otras comunidades desérticas, independiente de su hábito y/o sistema radicular (Reynolds et al. 1999).

Basado en estos resultados se postula que una disminución en las precipitaciones anuales, como las observadas en la pluviometría de La Serena en los últimos 100 años, de valores promedios cercanos a $170 \mathrm{~mm}$ a principios del siglo XX, hasta valores bajo los $80 \mathrm{~mm}$ en los últimos 30 años (Santibáñez 1997, Squeo et al. 1999, Novoa \& López 2001) resultarían en una disminución paulatina en la frecuencia de especies altamente dependientes de la humedad superficial como $B$. peduncularis, mientras que la frecuencia de especies capaces de explotar fuentes de agua subterránea como $H$. parvifolius se vería menos afectada. Lamentablemente, la alta dependencia a humedad superficial exhibida por $B$. peduncularis parece ser compartida por otras especies nativas con interés forrajero (Squeo et al. 1999, 2000). Frente a un escenario climático en el que las precipitaciones continuaran disminuyendo (CONAMA 1999), la menor disponibilidad de agua superficial esperada resultaría en una disminución de la productividad y diversidad de las especies nativas con interés forrajero en el secano costero del norte-centro de Chile.

\section{AGRADECIMIENTOS}

Este trabajo está basado en la tesis de Ingeniería Agronómica del primer autor. Los autores agradecen a Julio Gutiérrez y a dos revisores anónimos por sus valiosos comentarios al manuscrito. Agradecemos el apoyo logístico dado por la Compañía Minera del Pacífico, faenas El Romeral, en especial a los Srs. Mario Rojo y Vasco Larraechea; y del Observatorio Inter-Americano Cerro Tololo.
Este trabajo fue financiado por los proyectos FONDECYT 1960037 y 1000035.

\section{LITERATURA CITADA}

ARAVENA R \& E ACEVEDO (1985) The use of environmental isotopes oxygen-18 and deuterium in the study of water relations of Prosopis tamarugo Phil. En: Habit MA (ed) The current state of knowledge on Prosopis tamarugo: 251-256. Food and Agriculture Organization, Santiago, Chile.

ARROYO MTK, FA SQUEO, J ARMESTO \& C VILLAGRÁN (1988) Effects of aridity on plant diversity in the northern Chile Andes. Annals of the Missouri Botanical Garden 75: 55-78.

CALDWELL MM \& JH RICHARDS (1990) Competing roots systems: morphology and models of absorption. En: Givnish TJ (ed) On the economy of plant form and function: 251-273. Cambridge University Press, Cambridge, United Kingdom.

CALDWELL MM, TE DAWSON \& JH RICHARDS (1998) Hydraulic lift: consequences of water effux from the roots of plants. Oecologia 131: 151-161.

CODY ML (1986) Roots in plant ecology. Trends in Ecology and Evolutuion 1: 76-78.

CONAMA (1999) Primera comunicación nacional bajo la Convención Marco de las Naciones Unidas sobre el Cambio Climático. Comisión Nacional del Medio Ambiente, Santiago, Chile. $153 \mathrm{pp}$

COLEMAN MC, TJ SHEPHERD, JJ DURHAM, JD ROUSE \& GR MOORE (1982) Reduction of water with zinc for hydrogen isotope analysis. Analytical Chemistry 54: 993-995.

DAWSON TE \& JR EHLERINGER (1991) Streamside trees that do not use stream water. Nature 350: 335 337.

EHLERINGER JR \& HA MOONEY (1983) Productivity of desert and mediterranean-cliimate plants. En: Lange OL, PS Nobel, CB Osmond \& H Ziegler (eds) Physiological plant ecology 4: 205-231. SpringerVerlag, Berlin, Germany.

EHLERINGER JR \& CB OSMOND (1989) Stable isotopes. En: Pearcy RW, JR Ehleringer, HA Mooney \& PW Rundel (eds) Plant physiological ecology field methods and instrumentation. Chapman and Hall Ltd., London, United Kingdom.

EHLERINGER JR \& CS COOK (1991) Carbon isotope discrimination and xylem $\mathrm{D} / \mathrm{H}$ ratios in desert plants. Proceedings of an International Symposium on the Use of Stable Isotope in Plant Nutrition, Soil Fertility, and Environmental Studies: 489-497. International Atomic Energy Agency, Wien, Ostereich.

EHLERINGER JR \& TE DAWSON (1992) Water uptake by plants: perspectives from stable isotope composition. Plant, Cell and Environment 15: 10731082.

EHLERINGER JR, SL PHILLIPS, WFS SCHUSTE \& DR SANDQUIST (1991) Differential utilization of summer rains by desert plants: implications for competition and climate change. Oecologia 88: 430434. 
FLANAGAN LB \& JR EHLERINGER (1991) Stable isotope composition of stem and leaf water: applications to the study of plant water-use. Functional Ecology 5: 270-277.

FLANAGAN LB, JR EHLERINGER \& JD MARSHALL (1992) Differential uptake of summer precipitation among co-ocurring trees and shrubs in a pinyonjuniper woodland. Plant, Cell and Environment 15: 831-836.

GAJARDO R (1994) La vegetación natural de Chile: clasificación y distribución geográfica. Editorial Universitaria, Santiago, Chile. 165 pp.

GIBSON AC \& PS NOBEL (1986) The cactus primer. Harvard University Press, Cambridge, Massachusetts. $296 \mathrm{pp}$.

GREGG J (1991) The differential ocurrence of the mistletoe, Phoradendron junisperium, on its host, Junisperus osteosperma in the western United States. M.Sc. Thesis, University of Utah, Salt Lake City, Utah. 78 pp.

GUTIÉRREZ JR (1993) Desertification effects on ephemeral plants in the Chilean coastal desert. Revista Chilena de Historia Natural 66: 337-344.

GUTIÉRREZ JR (2001) Dynamics of ephemeral plants in the coastal desert of north-central Chile. En: Prakash I (ed) Ecology of desert environments: 105-124. Scientific Publishers, Jodhpur, India.

HADLEY NF \& SR SZAREK (1981) Productivity of desert ecosystems. BioScience 31: 747-753.

HAGERMANN R, G NIEF \& E ROTH (1970) Absolute isotopic scale for deuterium analysis of natural waters: absolute D/H ratios for SMOW. Tellus 23: 712-715.

LIN G, SL PHILLIPS \& JR EHLERINGER (1996) Monsoonal precipitation responses of shrubs in a cold desert community on the Colorado Plateau. Oecologia 106: 8-17.

LYNCH J (1995) Root architecture and plant productivity. Plant Physiology 109: 7-13.

NOVOA JE \& D LÓPEZ (2001) IV Región: el escenario geográfico físico. En: Squeo FA, G Arancio \& JR Gutiérrez (eds) Libro rojo de la flora nativa y de los sitios prioritarios para su conservación: Región de Coquimbo: 13-28. Ediciones Universidad de La Serena, La Serena, Chile.

NOY-MEYER I (1973) Desert ecosystem: environment and producers. Annual Review of Ecology and Systematics 4: 25-51.

OLIVARES S \& FA SQUEO (1999) Patrones fenológicos en especies arbustivas del desierto costero del nortecentro de Chile. Revista Chilena de Historia Natural 72: 353-370.
RADA F, FA SQUEO, A AZÓCAR \& HM CABRERA (1999) Water and carbon relations in the genus Adesmia DC. (Papilionaceae) at different altitudes in the high north-central Chilean Andes. Revista Chilena de Historia Natural 72: 201-211.

REYNOLDS JF, RA VIRGINIA, PR KEMP, AG DE SOYZA \& DC TERMMEL (1999) Impact of drought on desert shrubs: effects of seasonality and degree of resource island development. Ecological Monographs 69: 60-106.

SANTIBÁÑEZ F (1997) Tendencias seculares de la precipitación en Chile. En: Soto G \& F Ulloa (eds) Diagnóstico de la desertificación en Chile: 31. Corporación Nacional Forestal, La Serena, Chile.

SCHWINNING S \& JR EHLERINGER (2001) Water use tradeoffs and optimal adaptations to pulse driven arid ecosystems. Journal of Ecology 89: 464-480.

SCHWINNING S, K DAVIS, L RICHARDSON \& JR EHLERINGER (2002) Deuterium enriched irrigation indicates different forms of rain use in shrub/grass species of the Colorado Plateau. Oecologia 130: 345 355.

STEUDLE E (2000) Water uptake by plant rotos: an integration of views. Plant and Soil 226: 45-56.

STEUDLE E (2001) The cohesion-tension mechanism and the acquisition of water by plant roots. Annual Review of Plant Physiology and Plant Molecular Biology 52: 847-875.

SQUEO FA, N OLIVARES, S OLIVARES, A POLLASTRI, E AGUIRRE, R ARAVENA, C JORQUERA \& JR EHLERINGER (1999) Grupos funcionales en arbustos desérticos del norte de Chile, definidos sobre la base de las fuentes de agua utilizadas. Gayana Botánica (Chile) 56: 1-15.

SQUEO FA, N OLIVARES, A VALENZUELA, A POLLASTRI, E AGUIRRE, R ARAVENA, C JORQUERA \& JR EHLERINGER (2000) Fuentes de agua utilizadas por las plantas desérticas y su importancia en planes de manejo y restauración ecológica. Boletín de la Sociedad Botánica de México 65: 95 106.

VALENTINI R, GE SCARASCIA-MUGNOSSA \& JR EHLERINGER (1992) Hydrogen and carbon isotope ratios of selected species of a Mediterranean macchia ecosystem. Functional Ecology 6: 627-631.

WHITE JWC, ER COOK, JR LAWRENCE \& WS BROECKER (1985) The D/H ratios of sap in trees: implications for water sources and tree ring $\mathrm{D} / \mathrm{H}$ ratios. Geochimica et Cosmochimica Acta 49: 237246.

WILLIAMS DG \& JR EHLERINGER (2000) Intra- and interspecific variation for summer precipitation use in pinyon-juniper woodlands. Ecological Monographs 70: $517-537$ 